\title{
Behavioral risk compensation and the efficacy of nonpharmacological interventions
}

\author{
Oliver Kacelnik ${ }^{1 \star}$ and Alex Kacelnik ${ }^{2}$ (D) \\ ${ }^{1}$ Department of Infection Prevention and Preparedness, Norwegian Institute of Public Health, Oslo, \\ Norway and ${ }^{2}$ Department of Zoology, University of Oxford, Oxford, UK \\ ${ }^{*}$ Correspondence to: Department for Infection prevention and preparedness, Norwegian Institute of Public \\ health, Oslo, Norway. E-mail: oliver.kacelnik@fhi.no
}

(Received 17 December 2020; accepted 22 December 2020; first published online 12 April 2021)

\begin{abstract}
The COVID-19 pandemic has forced governments around the world into drastic measures without the normal evidence base or analyses of consequences. We present a quantitative model that can be used to rapidly assess the introduction and interaction of nonpharmaceutical infection prevention measures (NPI) both in rapid a priori predictions and in real-world $a$ posteriori evaluations. Two of the most popular NPIs are imposing minimum physical interpersonal distancing and the use of face coverings. The success of both measures is highly dependent on the behavior of the public. However, there is very little published information about the interactions between distance, mask wearing, and the behavioral adaptations that they are likely to generate. We explore the relation between these two fundamental NPIs and the behavioral responses that they may induce, considering both risk compensation and social norms enhancement. At present, we do not have the necessary information to parameterize our model to a sufficient degree to generate quantitative, immediately applicable, advice, but we explore a vast parameter space and illustrate how the consequences of such measures can range from highly beneficial to paradoxically harmful in plausible real situations.
\end{abstract}

Keywords: COVID-19; face masks; risk compensation; behavioral responses

\section{Introduction}

The severe acute respiratory syndrome coronavirus 2 (SARS-CoV-2) pandemic has led to some of the most widespread, drastic, and rushed public health measures the world has ever seen. Due to a lack of either a vaccine or effective therapeutics, authorities across the globe rely on nonpharmaceutical infection prevention measures (NPIs) in order to contain the virus. Under pressure for timely interventions, these measures are typically introduced hastily and inconsistently, often without the possibility for evidence-based decision-making, and, therefore, there is no alternative but to rely on $a$ posteriori observational analysis to determine their effectiveness. In fact, in many cases, it stricted re-use, distribution, and reproduction in any medium, provided the original work is properly cited. 
is not clear that decision makers and authorities are even aware of what kind of information they may need to predict the consequences of specific measures.

The most popular nonpharmaceutical NPIs are an increase in interpersonal distancing, the use of face covers, and handwashing. In each case, effectiveness is strongly dependent on the behavior of the public, but behavior is often overlooked and excluded from both predictive and a posteriori analyses. This may be partly due to the near absence of tools suitable to assess how the NPIs interact with the behavioral adaptations that they are likely to elicit. We present a quantitative framework aimed at exploring these relationships, considering that regulations can induce both risk-compensatory adaptations and social norms enhancement, depending on the circumstances. The information necessary to parameterize our framework to a degree that would allow it to generate immediately applicable predictions is not yet available, but we explore a vast parameter space and illustrate how the consequences of different measures can range from highly beneficial to paradoxically harmful in plausible real situations. We highlight what needs to be known to devise policies likely to achieve the desired consequences.

To anticipate behavioral responses is complex because the same regulation or advice can affect the public's behavior in opposite directions (Hedlund, 2000; Chu et al., 2020; Martin et al., 2020). On the one hand, it is known that people regulate the amount of risk they are willing to take through what is known as 'risk compensation'. This implies that individuals behave as if they had a 'risk thermostat': when a measure protects them from a given hazard without changing the setting of the thermostat, they modify their behavior so as to maintain the level of risk they are willing to tolerate. Evidence for such effects is particularly compelling in sport activities and in the behavior of car drivers: cyclists are more adventurous when induced to wear a helmet, and car drivers increase their speed when forced to wear seat belts (Lund Zador, 1984; Adams, 1995; Morrongiello et al., 2007; Rudin-Brown \& Jamson, 2013). On the other hand, there is also evidence showing that information that increases the salience of social norms can lead to changes in compliance. If people are informed of whether others are following or breaching a given norm, they adjust their own behavior (Bicchieri \& Xiao, 2009; Bicchieri, 2016; Civai \& Ma, 2017; Betsch et al., 2020), so that prescribing the use of face masks may bring the infection risk to the attentional fore and enhance interpersonal distancing. Such relations could explain outcomes in studies that found a positive correlation between NPIs such as mask wearing and personal distancing.

Overall, NPI effects are variable and likely to be highly dependent on the situation and demographic targeted. From the point of view of health authorities, it is the net effect when compensatory behavior is included that should inform the selection of a suitable NPI. However, behavioral factors are poorly researched and they are rarely integrated in the analyses. We use the term 'raw effect' for the risk reduction due to the implementation of an NPI in the absence of any other change in behavior (i.e., just wearing masks or washing hands without any other change in an individual's actions). In contrast, the 'net effect' includes the changes in risk when additional behavioral impacts are considered. It is important to establish the circumstances in which raw and net effects may be sufficiently different to warrant modification of the prevention strategy. 
The framework described below focuses on decision-making, a posteriori analysis, and justification of regulations regarding social distancing and face covering in the context of SARS-CoV-2, but the modeling strategy is applicable to other situations where the ultimate efficacy of NPIs depends on behavior. We start with a description of the expected interactions between social distancing and face mask wearing, and then develop a formal model that explicitly incorporates potentially critical behavioral adaptations that could affect prescriptive regulations.

The need for a framework suitable to integrate sources of variance in the efficacy of NPIs is evidenced by the conflicting outcomes observed across different types of implementation. Schünemann et al. (2020) express the problem thus:

Despite advances in pharmacological treatment and early vaccine development, reducing transmission of the virus with the use of facemasks (referring to medical or surgical masks, N-95 and similar respirators, cloth masks, and bandannas) by health-care workers and the public alike remains a hotly debated topic due to politicisation of discourse and decision making.

Hopefully, evaluating potential outcomes in a formal mathematical framework will explain some of the observed variance and increase the effectiveness of public health decisions.

\section{Distance}

Increasing distance between a source of infection and a potential new host reduces the risk. As we will show, however, the shape of the function describing the decline in transmission likelihood as a function of physical separation is crucial when it comes to predicting the impact of face-covering regulations. This function depends on the relative participation of particles of different size: larger particles (droplets $>5 \mu \mathrm{m}$ ) fall due to gravity, and their contribution declines more steeply with distance than that of smaller airborne particles (aerosols) that reach farther and lose impact as they dilute in larger volumes of air with greater distance from the source (Morawska \& Milton, 2020; CDC Scientific Brief, 2020). Both decline with distance, but the shape of the function is different. For our model, we assume that ceteris paribus, the probability of virus transmission between an infected and a healthy individual, declines following a sigmoid (inverted ' $S$ ') function of their separation. One reason to expect an inflection is the different behaviors of droplets and aerosols, with aerosols declining through dilution rather than gravity and taking greater relative responsibility beyond the range of reach of droplets. For any particular distancing and form of interaction, we assume that wearing a face mask reduces the probability of transmission by a multiplicative factor, the value of which depends on the quality of the mask and the correctness of use.

\section{Face covering}

The properties of different kinds of mask have a long history of being quantified in healthcare settings and as a public health measure, especially in Asia. However, masks 
are tested and specialized for different settings and needs. The protective qualities of FFP3, FFP2/N95, Type II, and Type I masks are relatively well documented in terms of their physical properties as barriers and when used by trained individuals. However, the pandemic has introduced commercial face masks and homemade face coverings into the mass market with little or no quality control, and it is fair to assume that in current real-life scenarios, there is a large diversity of face-covering devices with vast variation in efficacy (Greenhalgh et al., 2020). Physical performance as particle filters is readily obtainable, but it is not a fail-safe indicator of disease transmission prevention. In Norway, for example, it was estimated that, assuming a mask efficacy of $40 \%$ reduction of relative risk (RR) and low background prevalence, 200,000 people would need to wear face masks to prevent one new infection per week, but this is entirely dependent on very specific assumptions (Iversen et al., 2020; Schünemann et al., 2020).

\section{Relative versus absolute risk}

We characterize the efficacy of any configuration of parameters in terms of reduction in RR by a factor assumed to be representative of average properties of the face covers prevalent in the population of interest. In the absence of richer information, we will assume that the RR reduction factor for any specific kind of face mask is constant across the distance of interaction. This assumption may prove to be insufficient in the future. Reductions in absolute risk (AR), however, are more significant, and we estimate them by combining $R R$ reduction with distance-specific risk.

In summary, we examine how physical distance, mask wearing, and behavior interact to determine the potential effects of regulations prescribing social distancing and face covering. We aim at producing a tool for quantitative decision-making and a conceptual framework to identify the critical parameters that should inform evidence-based health regulations.

\section{Model and results}

Our model is based on the following assumptions:

When both an infected and a healthy person meet, the probability of contagion $C$ declines as a function of distance $D$, following the sigmoid relation implemented in the following equation:

$$
C(D)=\frac{C_{\max }+C_{\min } * D_{\frac{1}{2}}^{-k} * D^{k}}{1+D_{\frac{1}{2}}^{-k} * D^{k}},
$$

where

$C_{\max }$ : upper limit of the transmission probability. Intuitively, it is the probability of contagion in an interaction at close proximity, in the absence of mask wearing. When scaled from a pairwise interaction to group analyses, this number represents a statistical summary of many contributing factors, such as the proportion infected in the population, the demographics and cultural habits of the group in question, and the number and duration of interactions. 
$C_{\text {min }}$ : lower asymptote of the transmission probability. This parameter can be intuited as the risk of contagion when distance is sufficiently large for risk to approach the background risk in the environment, without a directional component.

$D_{1 / 2}$ : Distance at which the probability of transmission $C(D)$ is half way between $C_{\max }$ and $C_{\text {min. }}$.

$k$ : A parameter controlling the sensitivity of contagion to distance.

Using Equation (1), we model the expected effect of legislation imposing face covers. The expected change in RR is a function of the four parameters in Equation (1) $\left(C_{\max }, C_{\min }, D_{1 / 2}\right.$, and $\left.k\right)$ and two additional ones. First, a parameter $\alpha$, when multiplied by risk in the absence of mask wearing, yields a lower level of risk $(0 \leq \alpha \leq 1)$. In our model, the reduction in risk described by $\alpha$ is the raw effect. We use $\alpha$ to quantify the reduction in infection risk, which is dependent on, but not identical to, the mask specification based on the mask filtration rate of infective droplets and aerosols. However, we can take the mask specification as our source of information, treating a device certified to filter out $95 \%$ of infective particles as reducing transmission to $5 \%$ of the unprotected condition. Thus, mask efficacy equals $100^{\star}(1-\alpha)$; if a mask is certified (or claimed) to reduce transmission by $95 \%$, the value of $\alpha$ is $5 \%$. Second, a parameter $\beta$, which describes the change in social distance due to changes in behavior; if an individual who would typically keep a separation of $1 \mathrm{~m}$ changes to $0.8 \mathrm{~m}$ when wearing a mask, then $\beta=0.8$. Risk compensation, namely, a reduction in distancing when wearing a mask, occurs when $\beta<1$, while social norm enhancing occurs when the imposition of mask wearing elicits greater distancing, and then $\beta>1$.

We now express the net probability of infection at a given distance by including both the efficacy of the average device and the consequent behavioral response. For simplicity, and to make the transition to a population-level interpretation more direct, we do not distinguish whether both effects act through two or just one of the interacting subjects. Different cases can be readily incorporated into the model. We refer to the net probability of transmission given an obligatory mask-wearing normative as $C(D) \mid m$ and model it using the following equation:

$$
C(D) \mid m=\propto * \frac{C_{\max }+C_{\min } * D_{\frac{1}{2}}^{-k} *(\beta * D)^{k}}{1+D_{\frac{1}{2}}^{-k} *(\beta * D)^{k}}
$$

Equation (2) quantifies the net risk of infection when masks with a filtration rate of $100^{\star}(1-\propto)$ induce a behavioral distance modification response $\beta$. In this equation, $D$ is the physical distance that members of the target demographic group would typically keep in the absence of the obligatory face-covering normative.

Figure 1 shows a graphic version of the model for illustrative purposes.

The figure illustrates that the effect of interventions such as prescribing mandatory face covering is complex and can conceivably even result in the opposite of what is intended. This highlights the need for making explicit assumptions for each of the intervening factors. A first step is to identify a quantitative index to inform decisionmaking, and this requires choosing informative output variables. 


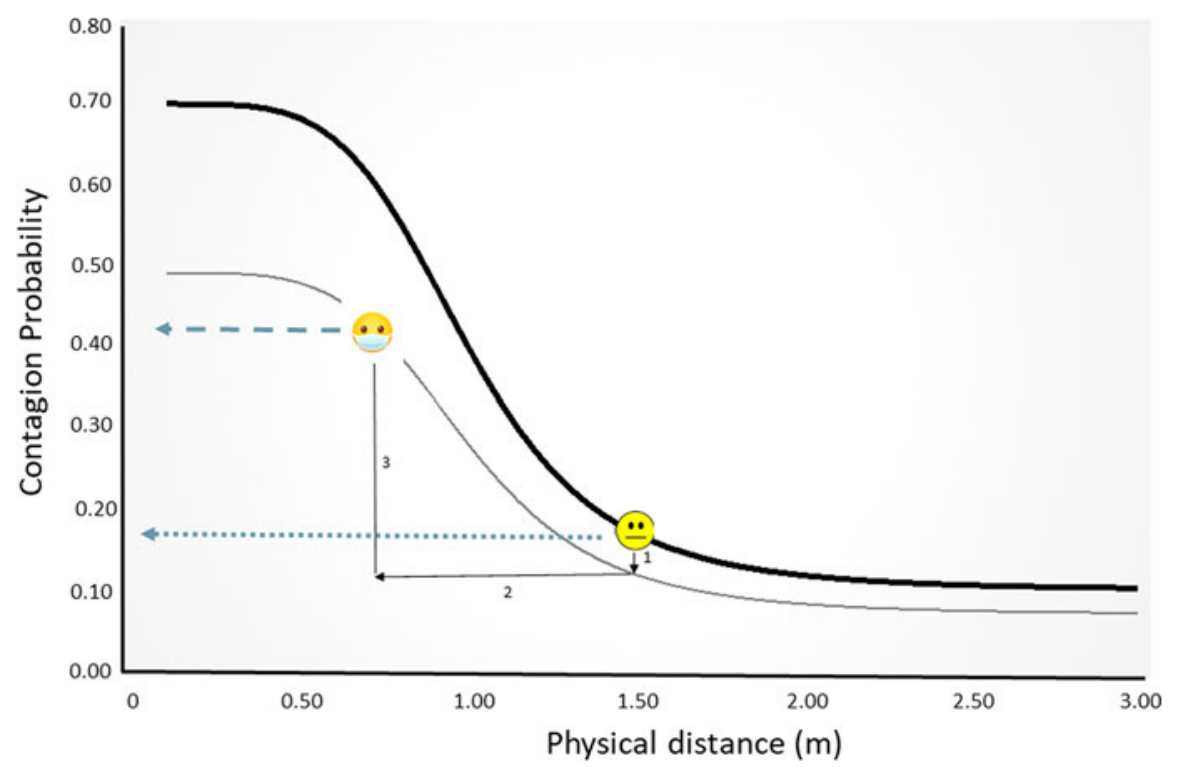

Figure 1. Interaction between distance, mask efficacy, and behavioral response, with illustrative parameters. For the figure, wearing a mask is assumed to reduce contagion at a given distance by $30 \%$ $(\alpha=0.7)$, and the change in physical distance is assumed to be a risk-compensatory reduction of $50 \%$ $(\beta=0.5)$. The thick black line shows the function describing the raw probability of contagion as a function of physical distance in the absence of any nonpharmaceutical intervention $\left(C_{\max }=0.7 ; C_{\min }=0.1 ; D_{1 / 2}=\right.$ $1 \mathrm{~m} ; k=5)$. The lighter thinner curve shows the corresponding function when wearing a mask with $30 \%$ efficacy. The interaction with behavior is shown for subjects that in the absence of any instructions would keep a distance of $1.5 \mathrm{~m}$. Solid arrows: (1) mask-mediated drop in contagion probability in the absence of risk compensation; (2) modification of physical distance due to a behavioral risk compensation of $50 \%$; (3) net risk after the intervention. The dotted black arrow points to raw risk in the absence of any intervention, and the dashed blue-gray one to net risk after both factors are considered. In this (illustrative) example, the behavioral response relative to mask quality is sufficient to result in a net risk increase. It is obvious that the relation between net and raw risk is dependent on the shape of the functions and the magnitude of the effects.

\section{$A R$ versus $R R$ changes}

Two indices of the effect of NPIs are RR and AR changes. We compute RR by expressing the probability of infection after both mask wearing and behavioral adjustment (Equation 2) as a fraction of raw risk (Equation 1), so that values below 1 are beneficial and those above are harmful.

$$
\text { Relative Risk Change }=\frac{C(D) \mid m}{C(D)}
$$

Changes in AR are of greater epidemiological significance and may be preferable to evaluate measures and communicate risk to the general public. This is given by the difference, rather than the ratio, between net and raw risk, expressed as a percentage, 
as follows:

$$
\text { Absolute Risk Change }=100 *[C(D) \mid m-C(D)]
$$

Figure 2 shows the effects of obligatory mask wearing in terms of relative (left) and absolute (right) risk changes for the parameters used in Figure 1. For the purpose of evaluating expected changes to inform decisions, the differences are substantial, and they highlight why the two metrics must be made transparent.

For the illustrative parameters that we used, the introduction of obligatory mask wearing has a beneficial effect on the wearers at short distances, is harmful around the distances that current legislation in most Western countries uses as guidance for distancing, and then declines in significance.

These illustrative results are not intended as ready-to-use quantitative estimates of the risk expected effect of a specific law or guideline, because they are dependent on our choice of arbitrary parameters. To further illustrate the variation in what might be expected, we show, in Table 1, a few illustrative cases.

The vast variety in potential changes highlights the importance of taking policy decisions with a suitably parameterized model that takes behavior into account and of communicating absolute as well as RR consequences. Once a model like the one presented here is available, the obstacle posed by uncertainty about specific parameter values can be mitigated by exploring the behavior of the model in as wide a parameter space as possible. The true efficacy of masks to prevent infection must be measured in the field, but mask rating can be used as a substitute, to assign a range of values to $\alpha$. For the behavioral responses, the task is more difficult because the impact of imposing obligatory protection measures could correlate positively or negatively with risk-taking responses. As mentioned earlier, obligatory mask use may lead some individuals to increase social separation, while others may shift to closer interactions than when uncovered.

In the next section, we keep fixed the relation between risk when unprotected and physical separation (using the parameters in Figure 1 to compute Equation 1) and explore the influence of $\alpha$ and $\beta$, the parameters representing mask efficacy and behavioral responses. We sample the range of mask efficacy across the possible range, in steps of $10 \%$, and explore behavioral responses ranging from extreme risk compensation, when obligatory mask wearing leads to reducing social distance to one-tenth of the original $(\beta=0.1)$ to cases where it leads to an increase in distancing of $50 \%(\beta=1.5)$. We repeat the calculations at four initial distances $(1,1.5,2$, and $3 \mathrm{~m}$ ), which cover the range used by many health authorities as guidance for protective social separation.

Figure 3 shows that when variation in mask efficacy and behavioral response are considered jointly, there exists regions of the $\alpha, \beta$ parameter space where imposing the use of masks has beneficial effects and regions where the net effects are harmful. The boundaries of these regions change drastically with the distance being considered. Further, the effect of distance on the sign of the effect can be nonmonotonic (see Figure 2), and the magnitude of these effects differs notably when relative or absolute changes in risk are considered. For variations in AR, effects when raw risk is low (at large physical distances) can be negligible. Our sensitivity analysis does 

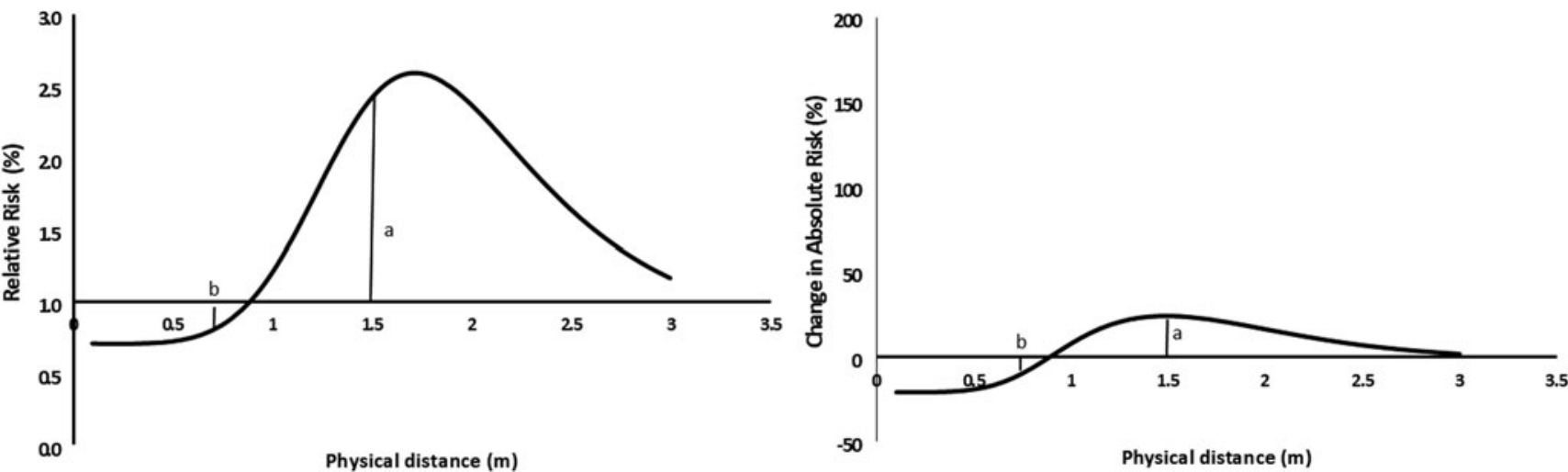

Physical distance $(\mathrm{m})$

Physical distance $(m)$

Figure 2. Relative (left) and absolute change in risk of contagion relative to raw risk, after regulatory introduction of mask wearing, computed with the illustrative parameters used in Figure 1. The physical distance in the abscissa indicates the social distancing for a given target group in the absence of intervention. The importance of communicating AR rather than RR is stressed by the vertical lines: the relative net effect (left) varies between a drop in contagion at a close proximity (b, $0.75 \mathrm{~m}$ ) of about $20 \%$ and an over twofold risk increase at intermediate distances (a, $1.5 \mathrm{~m}$ ). In contrast, the changes in absolute vary between a drop of roughly $12 \%$ (a) and an increase of $24 \%$ (b) across the same range. 
Table 1. Illustrative cases based on the risk versus distance function shown in Figure $1\left(C_{\max }=0.7, C_{\min }=\right.$ $\left.0.1, D_{1 / 2}=1, k=5\right)$. The bold cells show cases where the intervention may result in an increase in the net risk of infection.

\begin{tabular}{|c|c|c|c|c|c|}
\hline & Case 1 & Case 2 & Case 3 & Case 4 & Case 5 \\
\hline $\begin{array}{l}\text { Pre-NPI social distance during } \\
\text { pandemic }(\mathrm{m})^{1}\end{array}$ & 1 & 1.5 & 1.5 & 2 & 2 \\
\hline Pre-NPI risk of infection & $40 \%$ & $17 \%$ & $17 \%$ & $12 \%$ & $12 \%$ \\
\hline Mask protective performance ${ }^{2}$ & $70 \%$ & $50 \%$ & $70 \%$ & $70 \%$ & $99 \%$ \\
\hline Behavioral distancing response, $\beta^{3}$ & 0.50 & 0.50 & 0.50 & 0.50 & 0.50 \\
\hline Post-NPI social distance $(\mathrm{m})^{4}$ & 0.5 & 0.75 & 0.75 & 1 & 1 \\
\hline $\begin{array}{l}\text { Risk with mask if social distancing } \\
\text { is unaltered }\end{array}$ & $12 \%$ & $8 \%$ & $5 \%$ & $4 \%$ & $1 \%$ \\
\hline $\begin{array}{l}\text { Risk with mask at modified social } \\
\text { distancing }\end{array}$ & $20 \%$ & $29 \%$ & $18 \%$ & $12 \%$ & $4 \%$ \\
\hline Relative risk ${ }^{5}$ & 0.50 & 1.71 & 1.06 & 1.00 & 0.33 \\
\hline Net mask effect, AR (\%) & $-20 \%$ & $12 \%$ & $1 \%$ & $0 \%$ & $-11 \%$ \\
\hline
\end{tabular}

Notes:

${ }^{1}$ Social distancing typical of targeted demographic in the absence of NPI.

${ }^{2}$ Mass protective performance $=100(1-\alpha)$. This is lower than the specified filtering rate under lab conditions.

${ }^{3}$ Factor by which distancing changes in response to NPI.

${ }^{4}$ Social distancing when wearing mask.

${ }^{5}$ Net risk/pre-NPI risk.

not explore other significant factors, among which the most important is background raw risk. Equivalent proportional risk variations cause different modifications in AR when background prevalence differs, and the latter must be considered when evaluating a measure against its costs. In our model, background prevalence determines the upper and lower levels of transmission $\left(C_{\max }\right.$ and $\left.C_{\min }\right)$ and is modified through the shape of the effect of distance function.

Regarding the latter, and given the absence of precise quantitative information, we hypothesized the shape of the raw risk variation with distance, the mean quality of mask being used, and the magnitude and sign of the behavioral responses. For this reason, the results shown in our figures and table are not meant to be used on their own but to expose the need for fieldwork aimed at quantifying the relevant parameters for each situation. Our hypothetical transmission versus social distance function may well be inaccurate, but decision makers evaluating the potential consequences of public health measures ought to replace it and the remaining parameters by better estimates when available, rather than proceeding without a predictive model.

\section{Conclusion}

The net consequence of any nonpharmaceutical public health intervention cannot be divorced from the behavioral adjustments it causes. If, for instance, promulgating the obligatory use of face masks induces a particular section of the population to compensate for the perceived reduction in risk by relaxing other preventive measures 


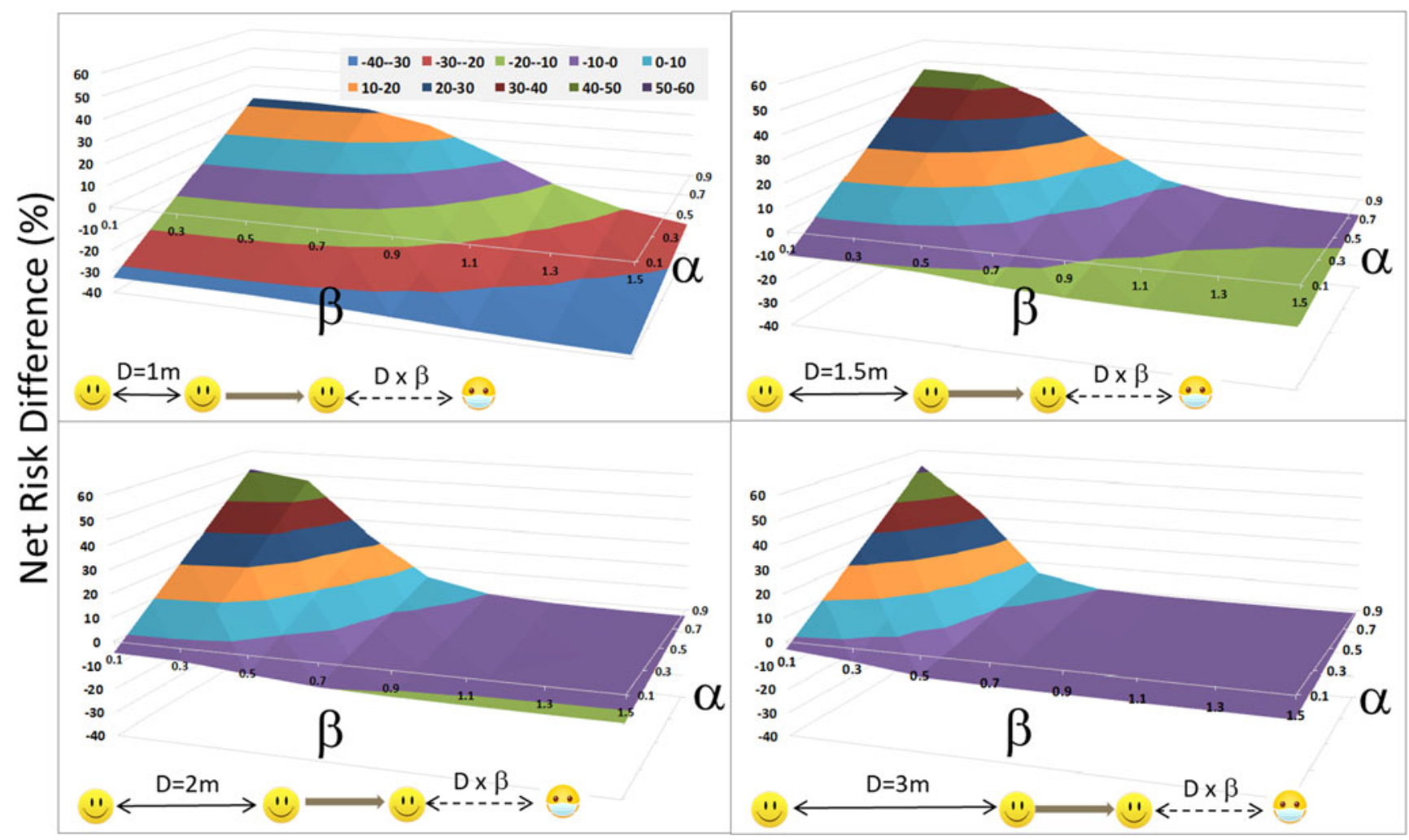

Figure 3. Net change in AR of contagion between unprotected (raw) interactions and those after the implementation of compulsory mask wearing, at four initial physical distances. The surfaces show the signed difference between the probability of contagion after and before the change. All four panels show net change as a percentage, for mask efficacy ranges from $90 \%(\alpha=0.1)$ to $10 \%(\alpha=0.9)$ and risk-compensation distance response from an extreme distance reduction $(\beta=0.1)$ to an enhancement of distancing of $50 \%(\beta=1.5)$. Raw risk parameters are the same as in Figure 1. Notice that the positive differences indicate a harmful increase in contagion probability. The greatest beneficial reduction in AR occurs at the front-right corner, when the mask blocks $90 \%$ of viral transmission, and obligatory mask wearing increases distancing by $50 \%$. Original physical separations are indicated by the emoji. 
such as distancing, physical contact, or handwashing, then the projected benefits can be far off the mark. Adding behavioral predictions is, thus, not optional but essential to take well-founded political decisions. Our model shows that under reasonable assumptions, interventions such as the obligatory use of face masks in public settings can have positive or negative consequences. This article demonstrates the importance of having a tool to make rational decisions based on quantitative approximations of the likely effects. Our implementation is based on functions and parameters that are not empirically validated, and for this reason, we do not intend to provide quantitative predictions but rather highlight what knowledge is necessary and inform what data should be extracted from previous experience. It is highly likely that riskcompensation behavior will differ greatly across demographic groups. For instance, sections of the population with a propensity to steep discounting of the future can well decide that risky activities such as attending a mass event fall below the risk threshold when wearing a mask, while the same category of person might have stayed at home otherwise. Other sections instead may be induced to substantially reduce their exposure if mask wearing acts as an enhancer of distancing norm compliance.

We anticipate that other authors may differ over the substantial decisions we have taken in shaping our model, particularly about the shape of the function relating the raw risk of transmission as a function of distance. We made our modeling choices based on reasoning about available information and would welcome better suggestions. We would consider our contribution successful if it served to promote further discussion using explicit hypotheses about the relevant interacting factors.

Acknowledgments. We are grateful to our colleagues Carlos Bernstein, Edward Mitchell, Adam Oliver and Hanne-Merete Eriksen-Volle for helpful comments on a previous version.

\section{References}

Adams, J. (1995), Risk: the policy implications of risk compensation and plural rationalities, London: Routledge; Taylor and Francis. 240 pages. ISBN-13 9781857280685.

Betsch, C., L. Korn, P. Sprengholz, L. Felgendreff, S. Eitze, P. Schmid and R. Böhm (2020), 'Social and behavioral consequences of mask policies during the COVID-19 pandemic', Proceedings of the National Academy of Sciences, 117(36): 21851-21853. doi:10.1073/pnas.2011674117.

Bicchieri, C. (2016), Norms in the wild. How to diagnose, measure, and change social norms, Oxford: Oxford University Press, 264 pages. ISBN 978-0190622053.

Bicchieri, C. and E. Xiao (2009), 'Do the right thing: but only if others do so', Journal of Behavioral Decision Making, 22(2): 191-208. doi:10.1002/bdm.621.

Centers for Disease Control and Prevention (2020), Scientific Brief: SARS-CoV-2 and Potential Airborne Transmission.Retrieved from: https:/www.cdc.gov/coronavirus/2019-ncov/more/scientific-brief-sarscov-2.html.

Chu, D. K., E. A. Akl, S. Duda, K. Solo, S. Yaacoub, H. J. Schünemann, and on behalf of the COVID-19 Systematic Urgent Review Group Effort (SURGE) study authors (2020), 'Physical distancing, face masks, and eye protection to prevent person-to-person transmission of SARS-CoV-2 and COVID-19: a systematic review and meta-analysis', Lancet, 395: 1973-1987. https://doi.org/10.1016/S0140-6736(20)31142-9.

Civai, C. and I. Ma (2017), 'The enhancement of social norm compliance: prospects and caveats', Journal of Cognitive Enhancement, 1: 26-30. https://doi.org/10.1007/s41465-017-0009-4.

Greenhalgh, T., X. H. Chan, K. Khunti, Q. Durand-Moreau, S. Straube, D. Devane, E. Toomey and A. Adisesh (2020), Retrieved from: https://www.cebm.net/covid-19/what-is-the-efficacy-of-standardface-masks-compared-to-respirator-masks-in-preventing-covid-type-respiratory-illnesses-in-primary-carestaff/. 
Hedlund, J. (2000), 'Risky business: safety regulations, risk compensation, and individual behaviour', Injury Prevention, 6: 82-90.

Iversen, B. G., D. F. Vestrheim, S. Flottorp, E. Denison and A. D. Oxman (2020), COVID-19: Should individuals in the community without respiratory symptoms wear facemasks to reduce the spread of COVID-19? [Covid-19: Bør personer i samfunnet bruke ansiktsmasker for å redusere spredningen av covid-19? Hurtigoversikt 2020], Oslo: Norwegian Institute of Public Health.

Lund, A. K. and P. Zador (1984), 'Mandatory belt use and driver risk taking', Risk Analysis, 4: 41-53. doi:10.1111/j.1539-6924.1984.tb00130.x.

Martin, G. P., E. Hanna and D. Dingwall (2020), 'Urgency and uncertainty: covid-19, face masks, and evidence informed policy', BMJ, 2020: 369. doi: https://doi.org/10.1136/bmj.m2017.

Morawska, L. and D. K. Milton (2020), 'It is time to address airborne transmission of COVID-19', Clinical Infectious Diseases, ciaa939. doi:10.1093/cid/ciaa939. Epub ahead of print. PMID: 32628269; PMCID: PMC7454469.

Morrongiello, B. A., B. Walpole and J. Lasenby (2007), 'Understanding children's injury-risk behavior: Wearing safety gear can lead to increased risk taking', Accident Analysis \& Prevention, 39(3): 618623. doi:10.1016/j.aap.2006.10.006. PMID 17112456.

Rudin-Brown, C. and S. Jamson (2013), Behavioural Adaptation and Road Safety: Theory, Evidence and Action, Boca Raton: CRC Press, 67. ISBN 9781439856673.

Schünemann, H. J., Schü, E. A. Akl, R. Chou, D. K. Chu, M. Loeb, T. Lotfi, R. A. Mustafa, I. Neumann, L. Saxinger, S. Sultan and D. Mertz (2020), 'Use of facemasks during the COVID-19 pandemic', The Lancet, 3: 2020. https://doi.org/10.1016/S2213-2600(20)30352-0

Cite this article: Kacelnik O, Kacelnik A (2022). Behavioral risk compensation and the efficacy of nonpharmacological interventions. Behavioural Public Policy 6, 1-12. https://doi.org/10.1017/bpp.2021.1 